\title{
ELECTRONIC LEVEL INDICATOR FOR THE REMOTE VERIFICATION OF WIND INSTRUMENT POSITION
}

\author{
R. J. SAND
}

h

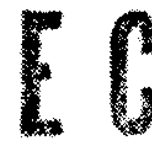

燥
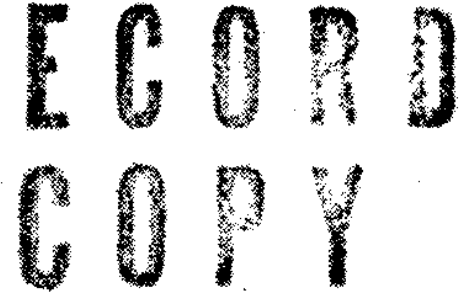

DO NOT RELEASE

FROM FILE

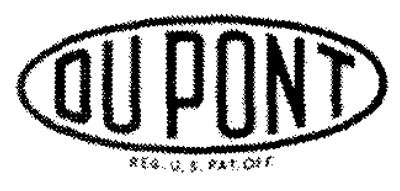

E. 1. du Pont de Nemours \& Co.

Savannah River Laboratory

Aiken, S. C. 29801

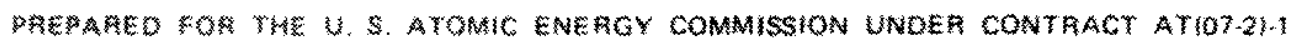




\section{NOTICE}

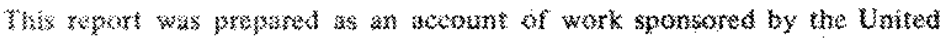

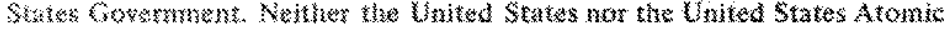

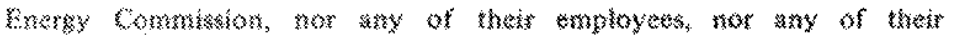

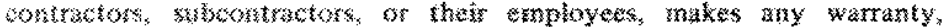

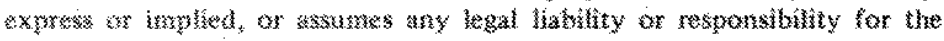

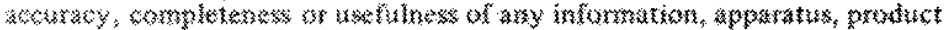
(2) (3)

Printed in the Wnited States of Annerica

Axatiable from

National wochricall hommation Service

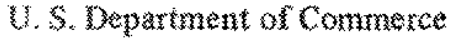

5285 pat aym

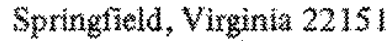

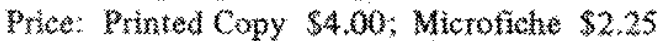


R. J. Sand

\section{Approved by}

D. E. Waters, Manager

Laboratory Operations and Services

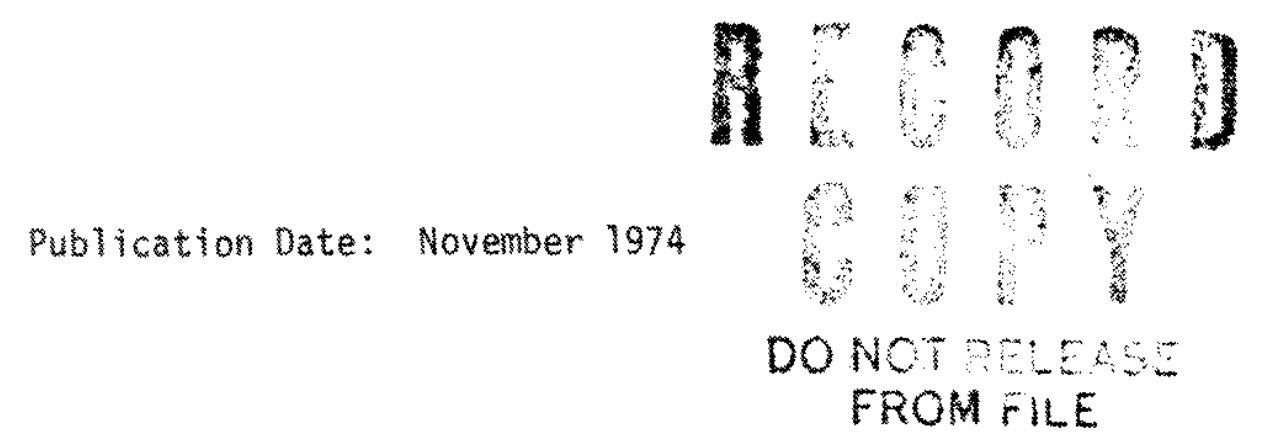

E. 1. du Pont de Nemours Co.

Savannah River Laboratory

Aiken. S. C. 29801 


\section{ABSTRACT}

A baterywowered, alectronic level indicatox was buj to

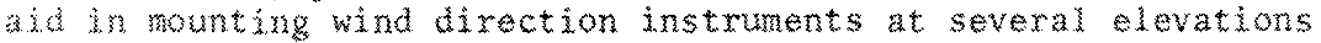
on a 365 minerer w tower. Two gravity-sersing transducers in each whd instrment are posicioned to indicate their orientation with respect to two horizontal axes, thereby determining the variation of the vertical drive shat from the zenth angle. Electrical signals from the tranducers axe transmitted through cables to a montoring station on the ground. 


\section{CONTENTS}

INTRODUCTION . . . . . . . . . . . . . . 7

INSTRUMENT DESCRIPTION . . . . . . . . . . . . . 7

Main Drive Unit.............. . . 11

Transducer Sensors . . . . . . . . . . 11

Power Pack . . . . . . . . . . . . 16

Ground-Level Monitoring Unit . . . . . . . . '16

SENSITIVITY . . . . . . . . . . . . . 16

REFERENCES . . . . . . . . . . . . . . 19 


\section{LIST OF TABLES AND FIGURES}

\section{Figure}

No.

1. Bivane Wind Direction Indicator . . . . . . . 8

2. Sensor Platform............... 9

3. Sensor Assembly . . . . . . . . . . . 10

4. Solid-State Main Drive Unit . . . . . . . . 11

5. Panel Face of Main Drive Unit . . . . . . . . . 12

6. Block Diagram of Circuitry . . . . . . . . 12

7. Main Drive Circuit ............. 14

8. Sensing and Zero-Test Circuits . . . . . . . 14

9. Amplifier and Rectifier Circuits . . . . . . 15

10. Meter Response Circuits . . . . . . . . . 15

11. Power Supply . . . . . . . . . . . . . . . 16

12. Ground-Level Monitor Unit . . . . . . . . . 17

13. Calibration of Electronic Level-Indicator . . . . 18

Tab1e

I. Calibration of Electronic Leve1-Indication. . . . 17 


\section{ELECTRONIC LEVEL INDICATOR \\ FOR THE REMOTE VERIFICATION \\ OF WIND INSTRUMENT POSITION}

\section{INTRODUCTION}

The Savannah River Laboratory (SRL) gathers data from wind instrumentation mounted on a local 365-meter television antenna tower. The degree of accuracy required for these data necessitates precise positioning of the wind direction instruments to obtain accurate azimuth and elevation measurements. Azimuthal corrections, if any, are noted by sighting on known landmarks and are mathematically incorporated in subsequent data analyses. Elevations are mechanically corrected to put data errors within tolerance. The wind instruments are mounted on booms fastened to the tower. Pitch and roll adjustments permit positioning of individual instmuments to indicate true wind direction.

During installation of the wind instruments, tower climbers encountered difficulties in final boom adjustment. Clamp-on bubble levels attached to secondary reference points were used to position the instruments, but sighting from ground-based telescopes indicated gross positional errors. Therefore, an electronic level indicator was developed to ascertain precise wind instrument position. Gravitysensing electrolytic transducers were mounted directly in the wind instruments, and their electronic nature allowed both tower climbers and ground personnel to observe and confirm the accuracy of installation.

\section{INSTRUMENT DESCRIPTION}

The wind direction instruments are Climet Bivane Wind Transmitters (Figure 1), manufactured by Climet Instruments Co., Sunnyvale, California. ' To obtain reliable information about the instrument position, it was necessary to mount the gravity-sensing transducers ${ }^{2,3}$ inside the bivane electronics housing. Because the vertical drive shaft of the bivane is securely attached to the base plate of the electronics frame, a suitable reference is provided to determine instrument position. The transducers are mounted on custom machined platforms to facilitate assembly of the modified bivanes. These platforms were machined and installed such that one transducer was parallel to the boom pitch axis, while the other was parallel to the boom roll axis (Figures 2 and 3 ). 


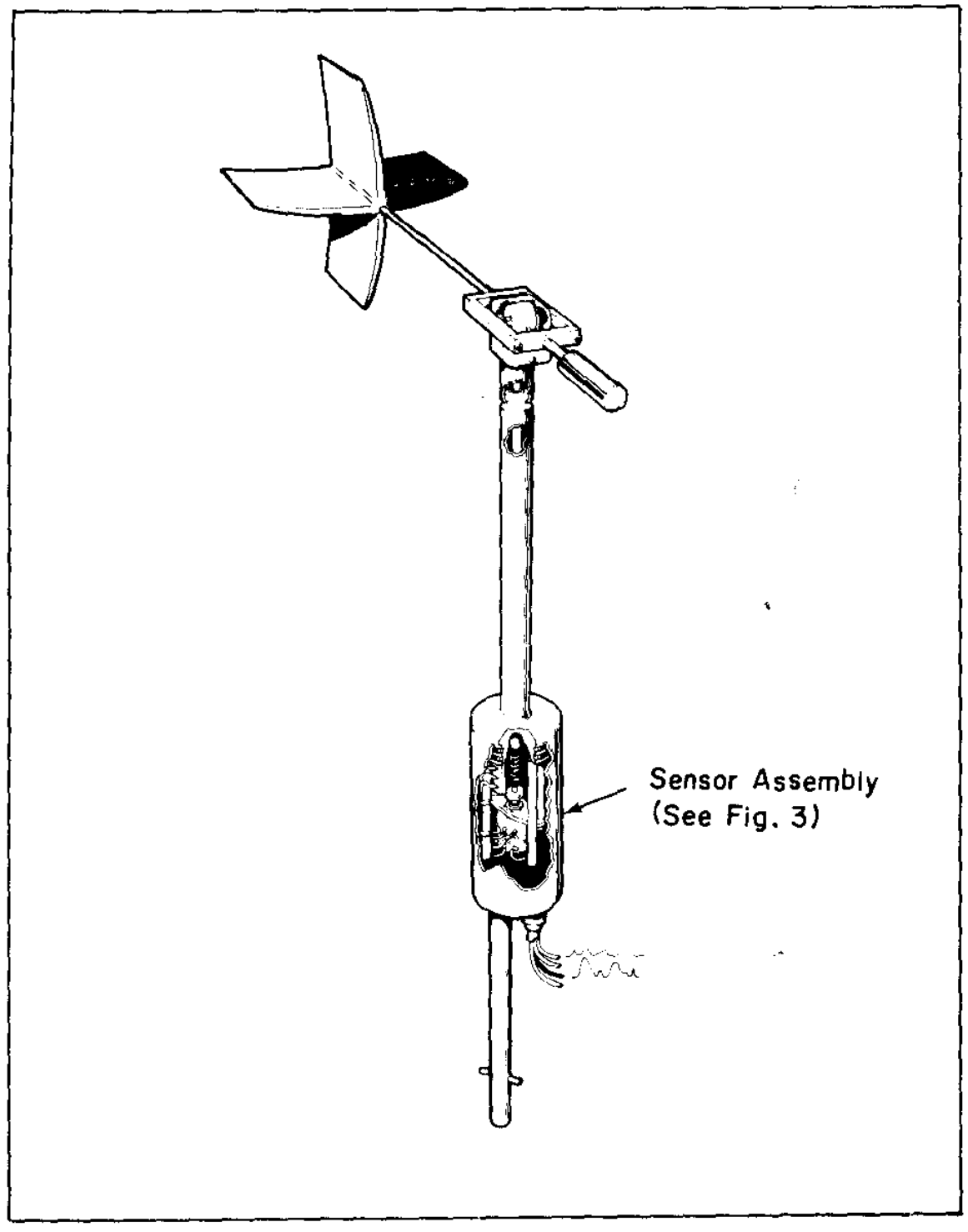

Figure 1. Bivane Wind Direction Indicator 


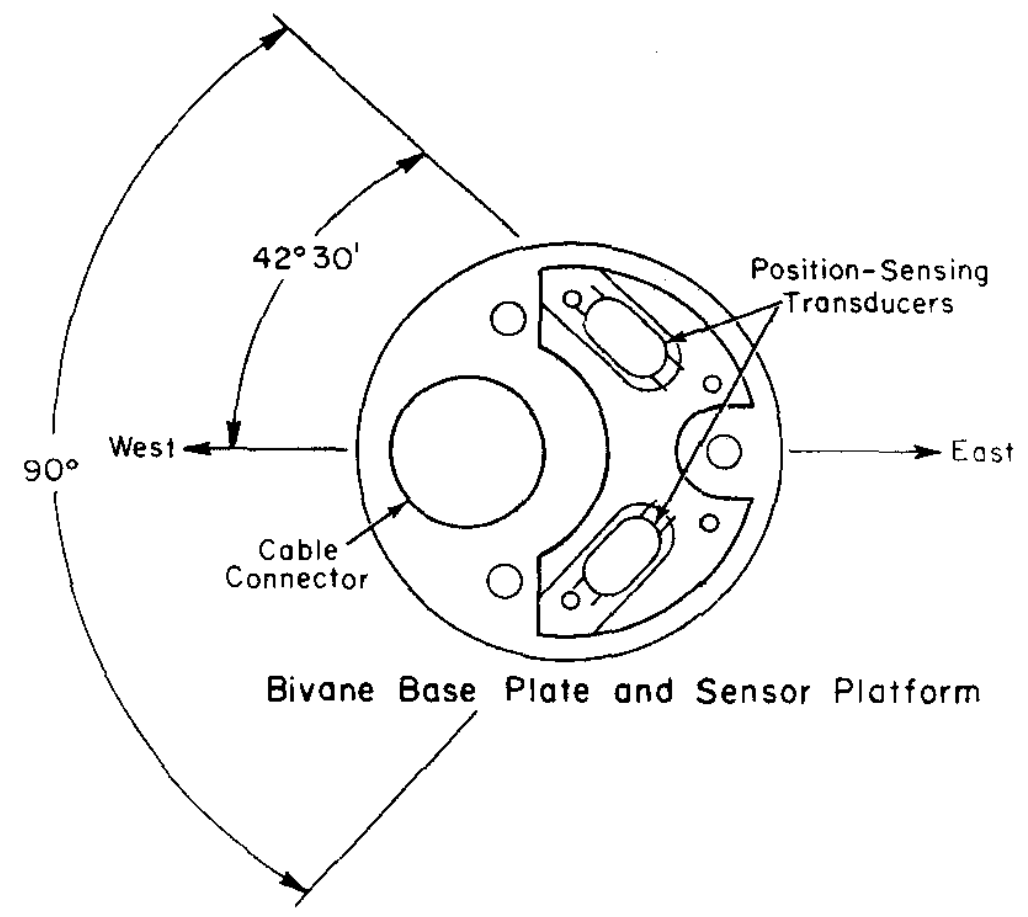

Figure 2. Top View of Sensor Platform 


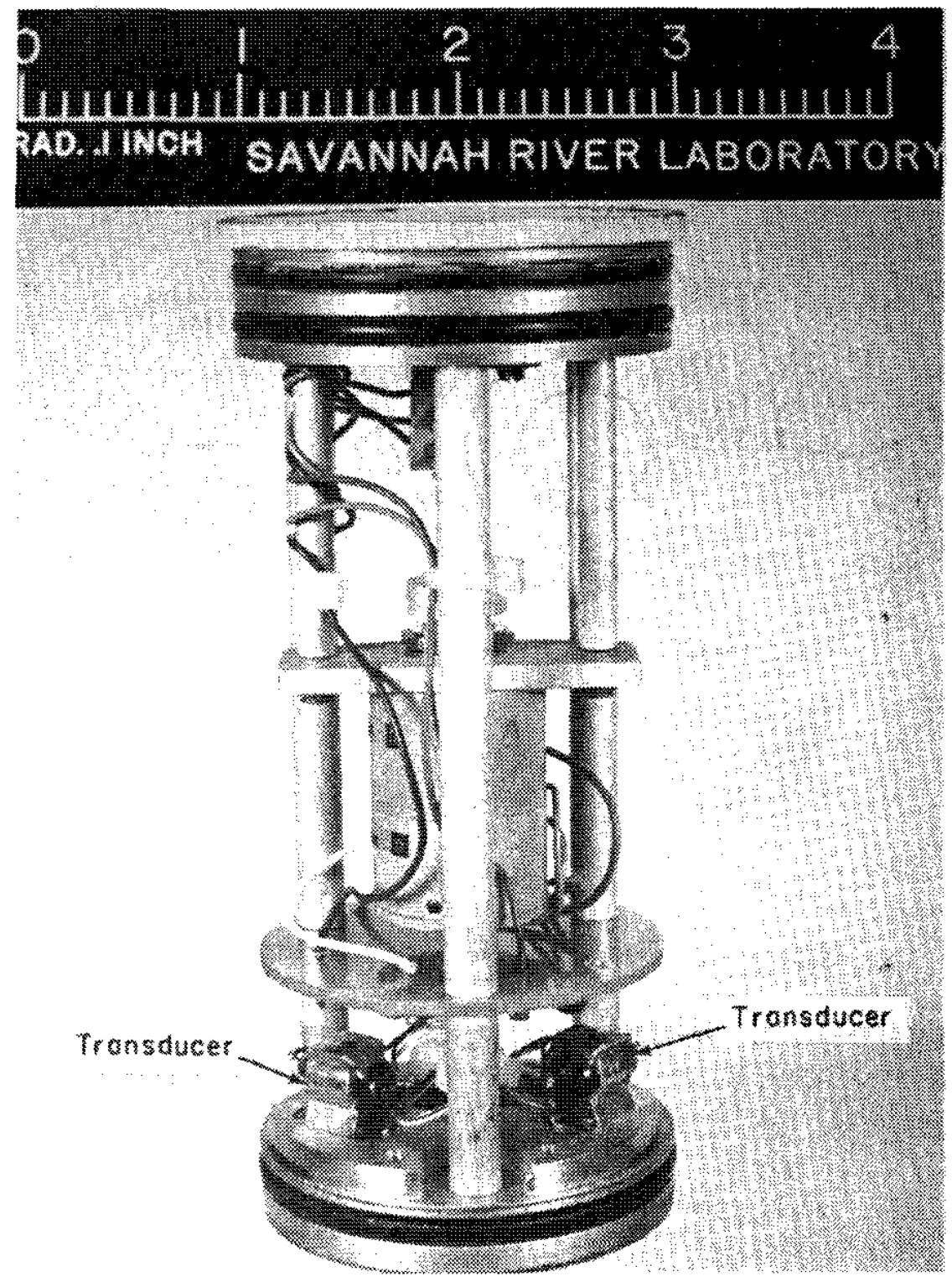

Figure 3. Sensor Assembly 


\section{MAIN DRIVE UNIT}

To reduce the weight of the instrument package to be taken up the tower by the tower climbers, a solid state unit was designed to drive and monitor the transducers (Figure 4). This instrument has circuitry to display position with respect to two axes simultaneously. Each detector has two five-position rotary switches (Figure 5) for coarse, medium, and fine sensitivity, and power supply voltage. The fifth position on each meter switch is marked "TRANS." This switch position electrically removes the meter from the circuit and shorts the terminals to minimize meter wear when not in use. Also included is a zero test button which replaces the transducer with a dummy load to simulate true leve 1 on the instrument.

\section{TRANSDUCER SENSORS}

The circuitry of the electronic level indicator can be divided into several blocks as shown in the block diagram (Figure 6).

Because of the electrolytic nature of the transducers, an $\mathrm{AC}$ power source is required to prevent chemically plating the electrolyte onto one electrode. A sinewave oscillator module ${ }^{4}$ was buffered with an amplifier, ${ }^{5}$ using a gain of one to drive the transducers (Figure 7). Each transducer operates as two potentiometers in series, with each half of the transducer changing resistance oppositely to the other half. That is, as one half increases resistance, the other half decreases resistance (Figure 8). The differential amplifiers are then placed across each half of the transducer. These amplifiers utilize a gain of two, because at true level, each amplifier sees half the driving voltage across the transducer (Figure 9). The output of each of these amplifiers is rectified (full-wave-positive) and is referenced to the battery ground.

The meter output is a measure of the voltage difference between the rectifier outputs to provide a proportional signal relative to the deviation from desired instrument position. Zerocenter microammeters converted to voltmeters allow directional deviation to be displayed (Figure 10). Each three-range voltmeter contains a fine, medium, and coarse leveling scale depending upon the degree of accuracy required. On the fine scale with \pm 25 divisions, each division is equivalent to an angle of 0.6 minute. 

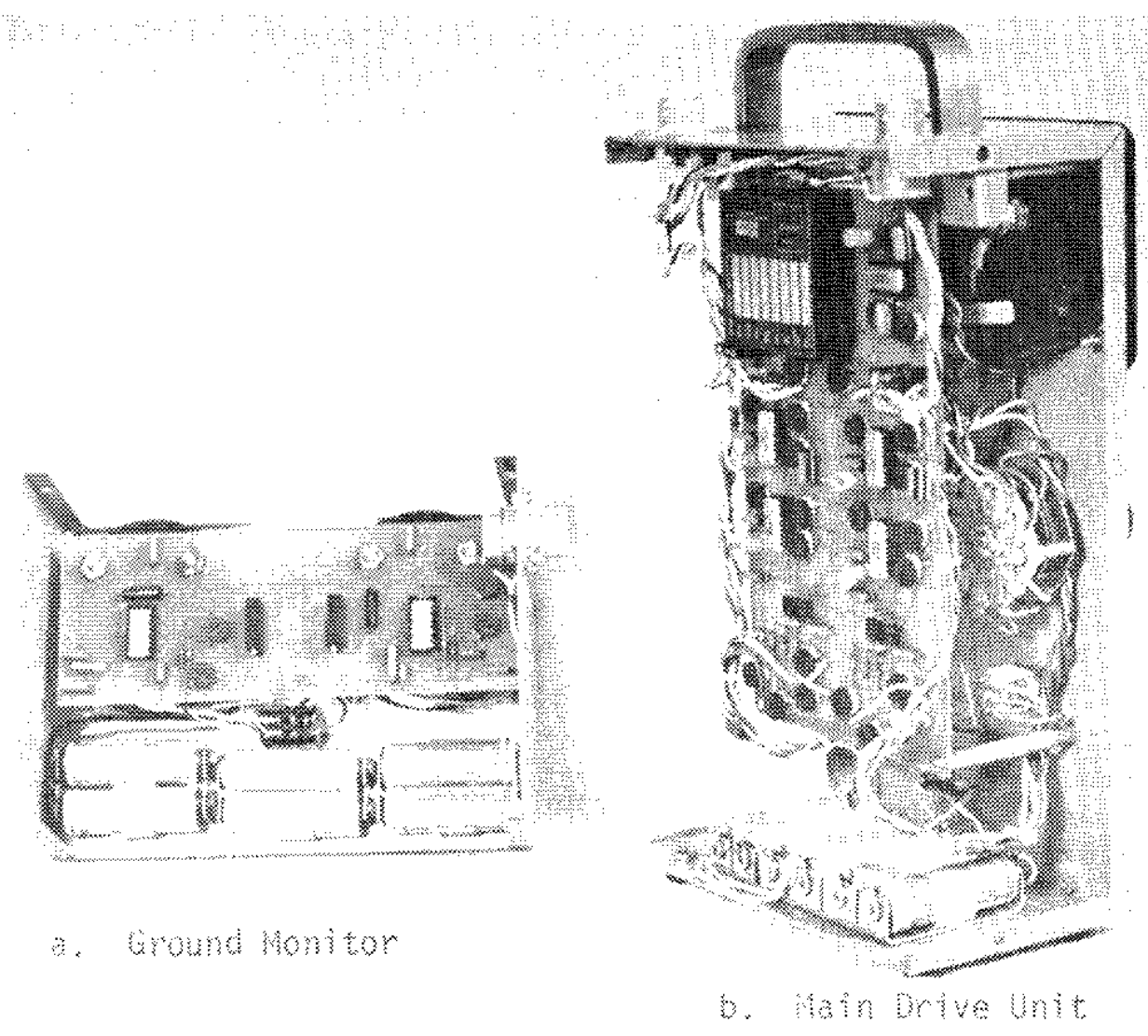

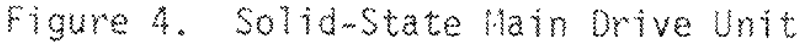
and Ground monitor 

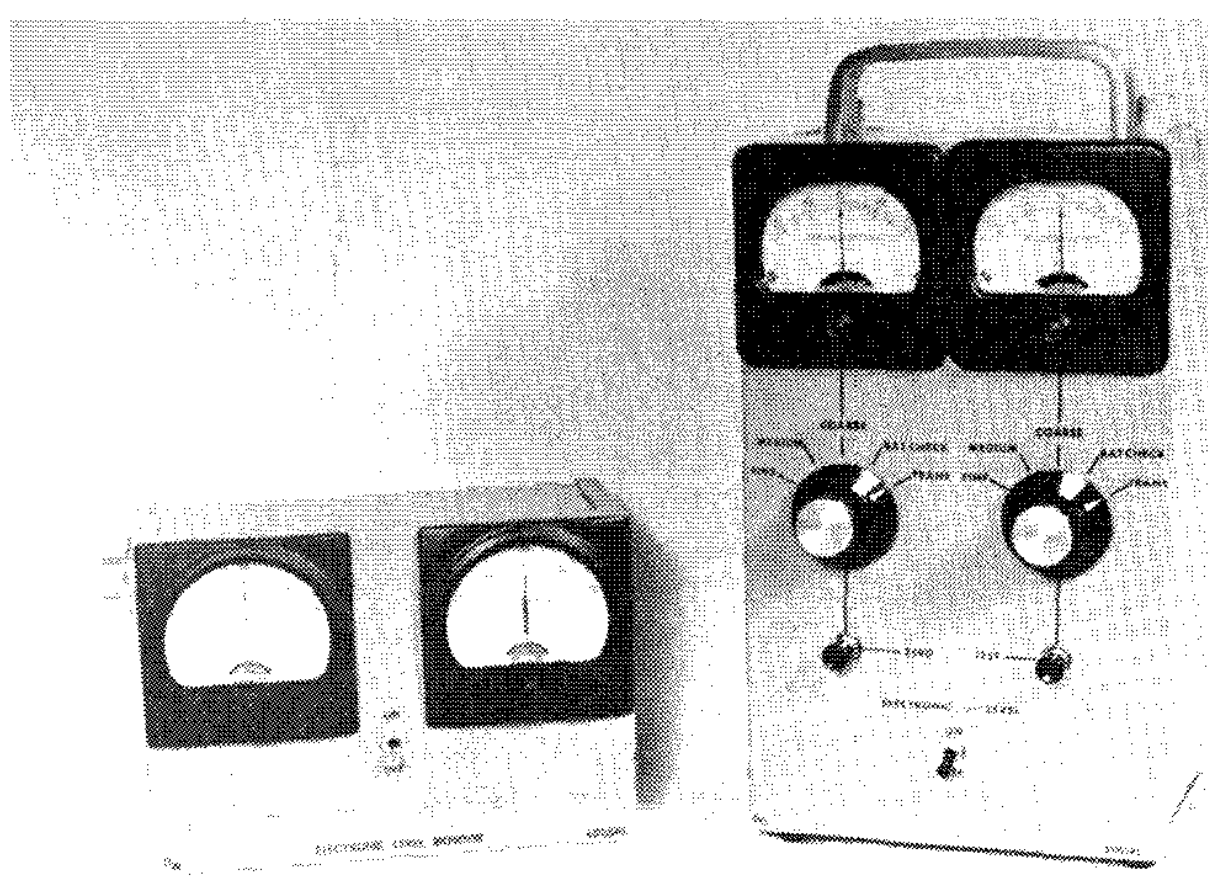

Figure 5. Panel Face of Main Drive Unit

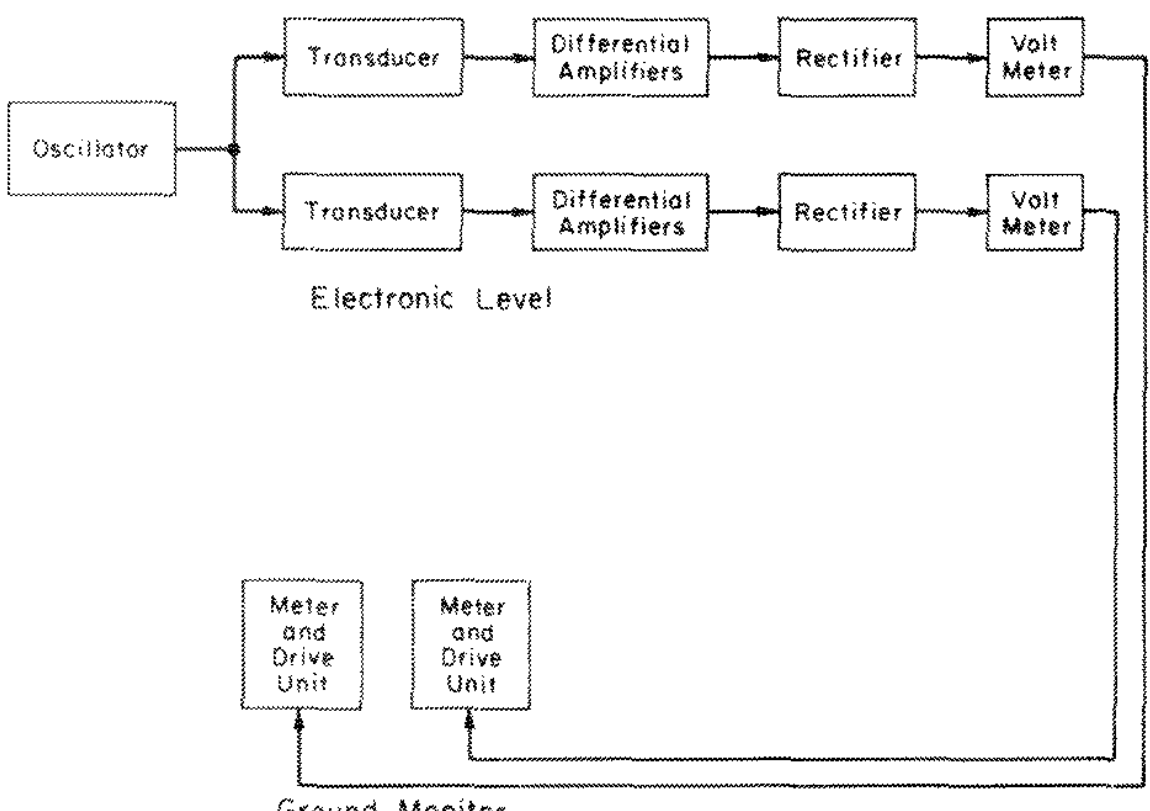

Ground Monitor

Figure 6. Block Diagram of Circultry 


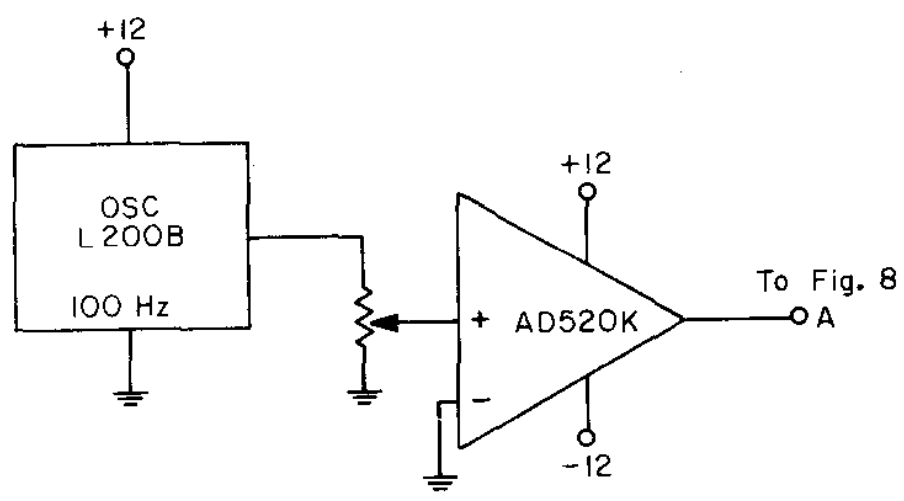

Figure 7. Main Drive Circuit

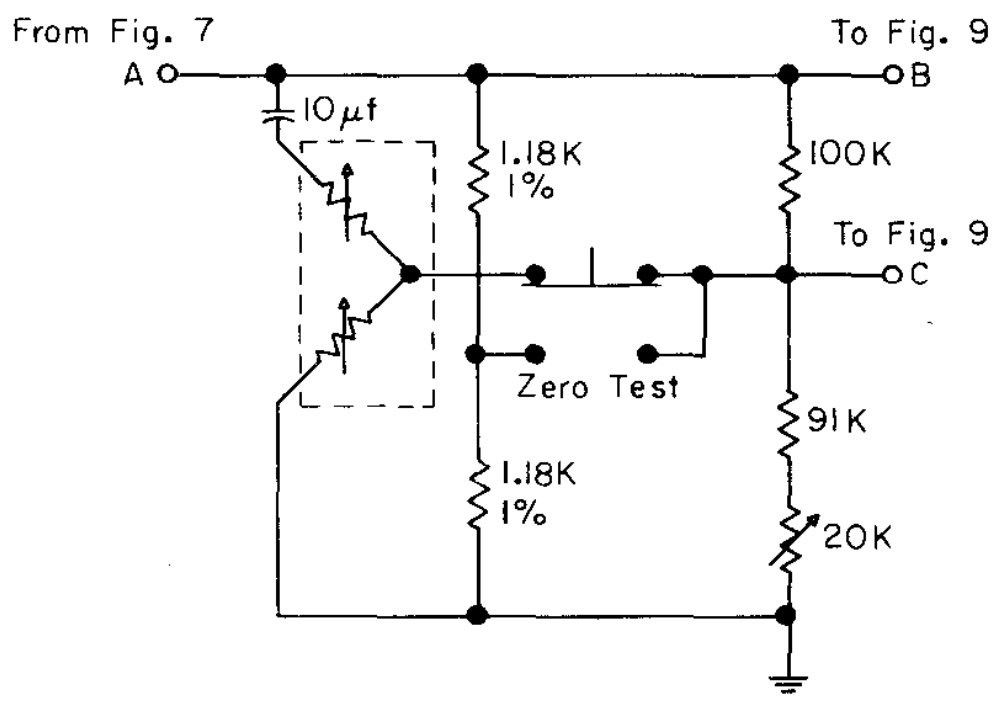

Figure 8. Sensing and Zero-Test Circuits 


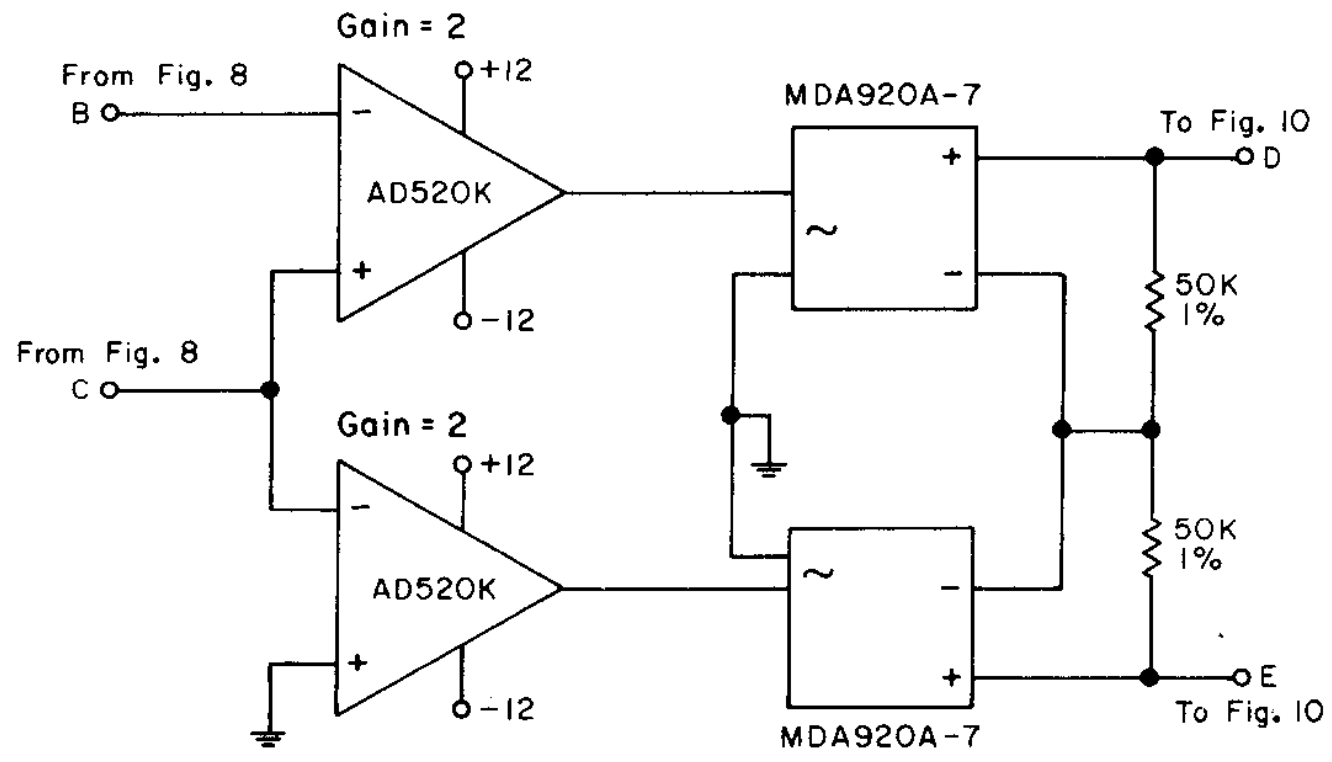

Figure 9. Amplifier and Rectifier Circuits

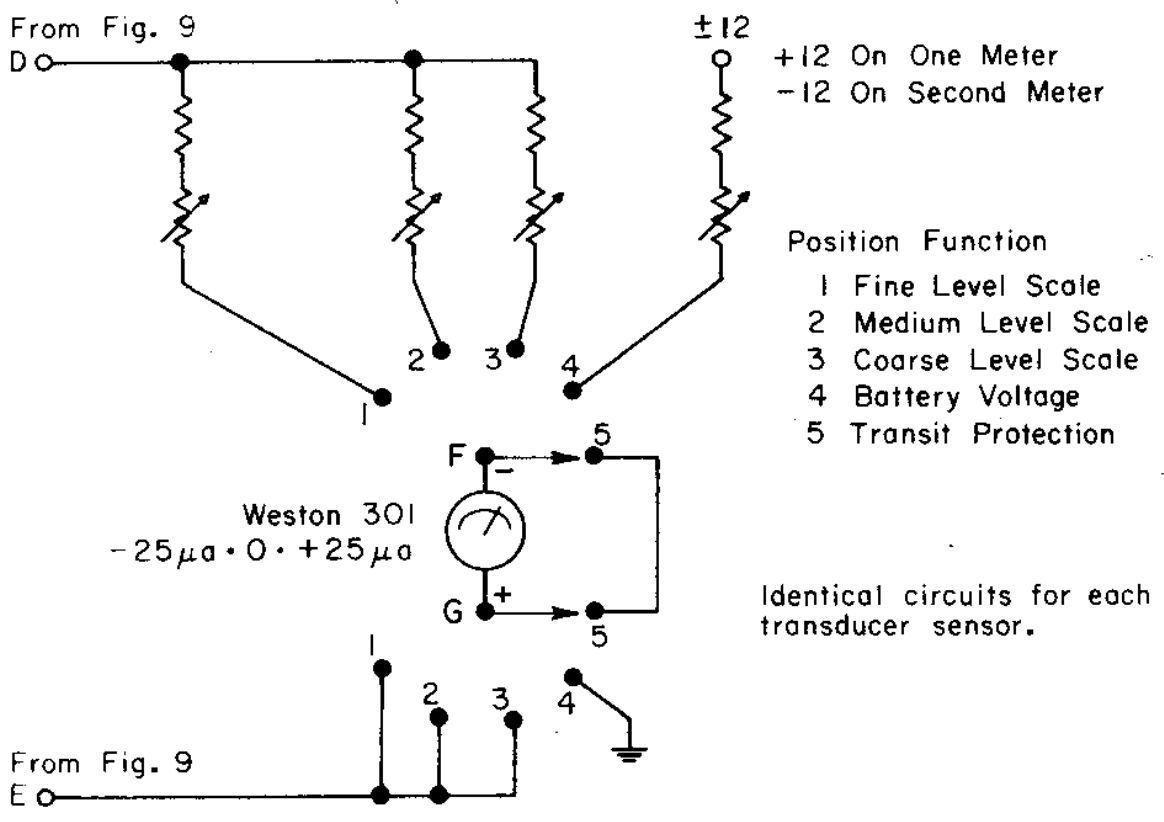

Figure 10. Meter Response Circuits 


\section{POWER PACK}

Included in the instrument are battery voltage test positions for the positive and negative power supply. Because of the portability requirements, battery power was necessary. Mercury batteries, because of their more desirable voltage discharge characteristics and onplant availability, were chosen to supply \pm 12 volts to the circuits (Figure 11).

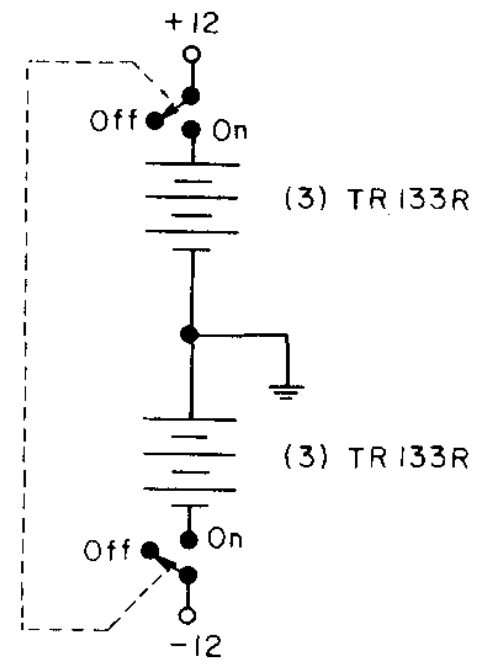

Figure 11. Power Supp Ty

\section{GROUND-LEVEL MONITORING UNIT}

As an added convenience to personnel observing the setup procedures of the instruments, a ground-level monitoring umit (Figure 12) was provided to permit observation of level indication without requiring this information to be relayed by radio from the tower climbers. The monitoring box was designed to monitor the meter outputs of the electronic level indicators at various tower elevations and to drive similar meters on the ground. Existing cables down the tower from each level connected the ground-level monitor to the main drive unit. Unfortunately, due to capacitance in the cables, the ground level unit did not operate when level indicators were above $91 \mathrm{~m}$.

\section{SENSITIVITY}

The level indicator has a sensitivity of 0.6 minute of angular displacement from the vertical, a factor of over 30 times better than the design criterium of 220 minutes. The indicator was cali- 
brated against a bubble level certified by the National Bureau of Standards. The response of the meters is shown in Table I and Figure 13.

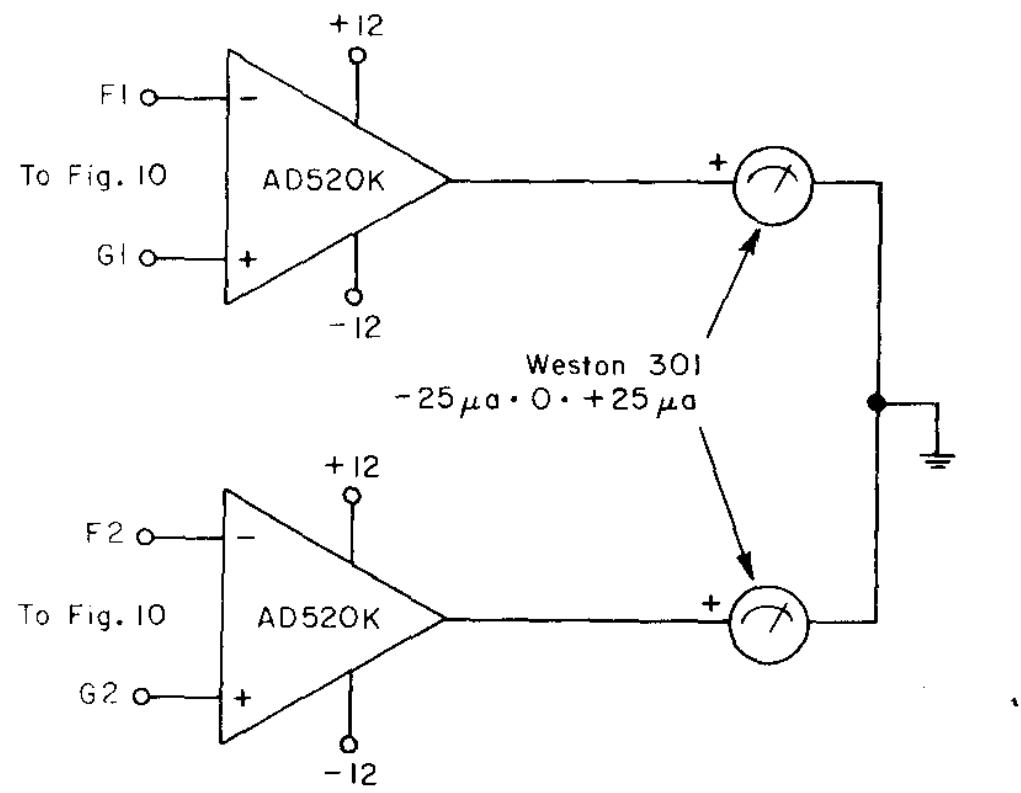

Figure 12: Ground-Leve] Monitor Unit

Table I. Calibration of Electronic Level Indicator

Reference Deflection, divisions

$-5$

$-4$

$-3$

$-2$

$-1$

0

1

2
Angular Meter Deflection, divisions

Change, min. Fine Medium Coarse

$-8.620$

$-6.896$

$-15.0$

$-3.25$

$-1.75$

$-11.0$

$-2.5$

$-5.172$

$-8.1 \quad-2.0$

$-5.5-1.1$

$-3.0-0.75$

$-1.724$

$0.0 \quad 0.0$

0.0

2.5

5.0 


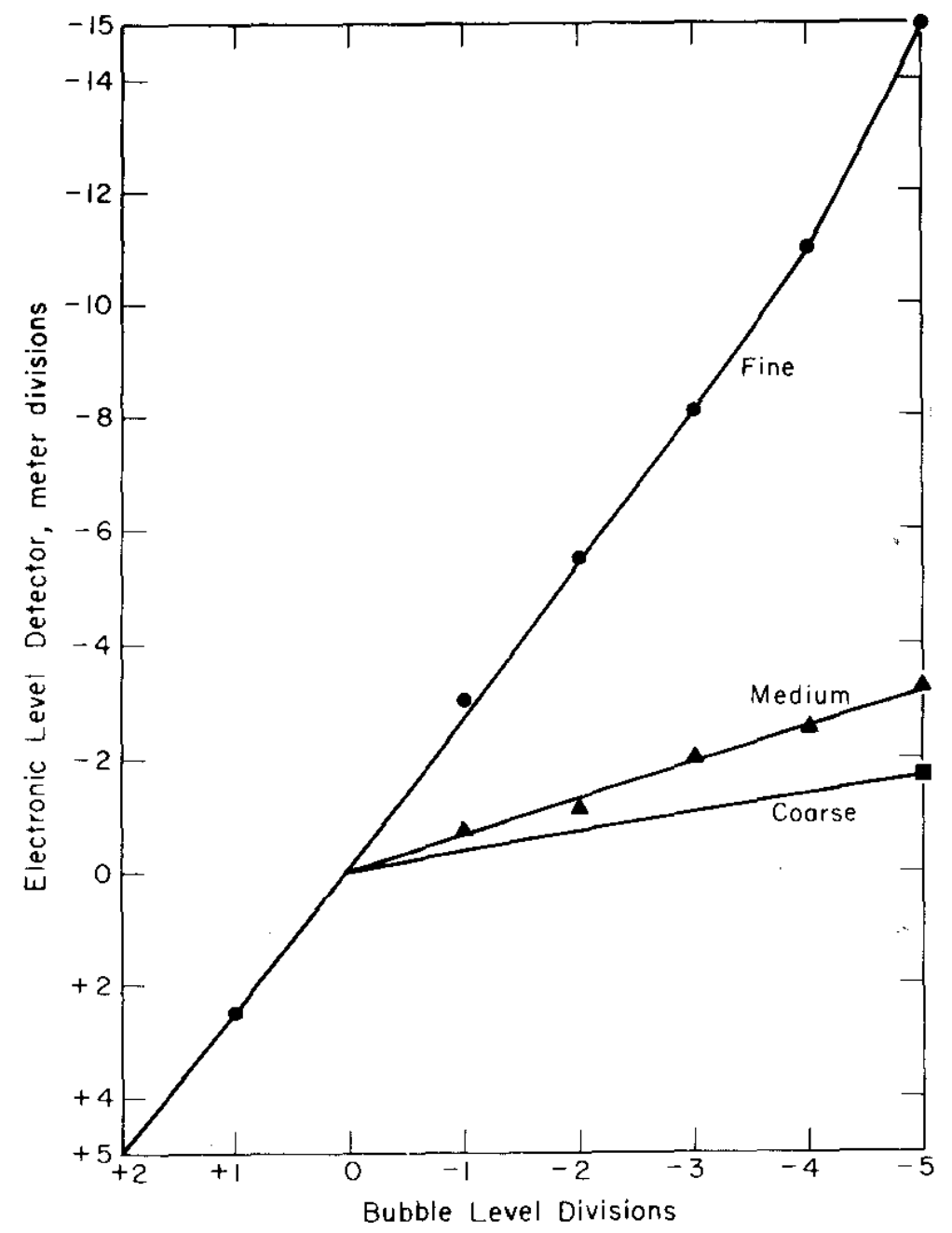

Figure 13. Calibration of Electronic Level Indicator 


\section{REFERENCES}

1. Bivane Wind Transmitter. Brochure 012-8A, -11. C1imet Instruments Co., Sunnyvale, California (1972).

2. C. E1derkin, D. C. Powe11, A. G. Dunbar, and T. W. Horst. Take-Off and Landing Critical Atmospheric Turbulence (TOLCAT)Experiments and Analysis. USAF Technical Report AFFDL-TR71-172. Air Force F1ight Dynamics Laboratory, Wright-Patterson Air Force Base, Ohio (1972).

3. Gravity Sensing Electrolytic Transducers. Brochure B-80000. Hamlin, Inc., Lake Mills, Wisconsin (1970).

4. PC Board Sinewave. Bulletin 70 F. Connor-Winfield Corp., Winfield, Illinois.

5. Integrated Circuit Instmmentation Amplifier - Preliminary Specifications. Brochure C129-20-5/72. Analog Devices, Norwood, Massachusetts (1972). 